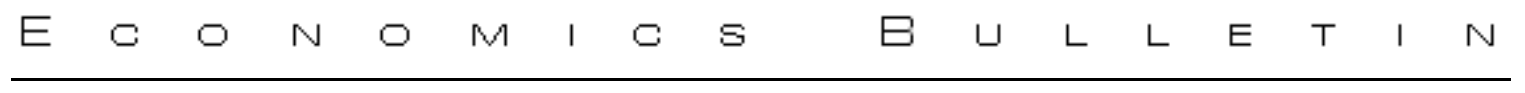

\title{
Fiscal policy and minimum wage for redistribution: an equivalence result
}

\author{
Arantza Gorostiaga \\ Universidad del País Vasco
}

\author{
Rubio-Ramírez Juan F. \\ Duke University and Federal Reserve Bank of Atlanta
}

\begin{abstract}
In this paper we derive conditions under which a minimum wage law combined with anonymous taxes and transfers and an agent-specific tax-transfer scheme are equivalent redistribution policies.
\end{abstract}

Financial support from Fundación Ramón Areces and SEC2003-02510/ECO is acknowledged. Beyond the usual disclaimer, we must note that any views expressed herein are those of the authors and not necessarily those of the Federal Reserve Bank of Atlanta or of the Federal Reserve System.

Citation: Gorostiaga, Arantza and Rubio-Ramírez Juan F., (2008) "Fiscal policy and minimum wage for redistribution: an equivalence result." Economics Bulletin, Vol. 5, No. 11 pp. 1-8

Submitted: November 6, 2007. Accepted: March 11, 2008.

URL: http://economicsbulletin.vanderbilt.edu/2008/volume5/EB-07E60012A.pdf 


\section{Introduction}

Many papers in the literature have considered the use of a minimum wage legislation jointly with a tax-transfer scheme to redistribute income among differently productive agents. Moreover, articles such as Allen (1987), Guesnerie and Roberts (1987) and Gorostiaga and Rubio-Ramírez (2004) have shown that minimum wages might be optimal when combined with linear taxes and transfers in a static general equilibrium model.

In this paper we prove that any equilibrium allocation attained under a minimum wage law and anonymous taxes and transfers could be implemented through an agent-specific tax-transfer scheme. We also show that the reverse implication is not always true. The equivalence depends on imposing some conditions on the initial agent-specific policy.

\section{The basic setup}

We consider a static general equilibrium model. The economy is populated by two types of agent: low and high productivity households. A proportion $\gamma$ of households are high-skilled $(H)$, and a proportion $1-\gamma$ are low-skilled $(L)$. This a production economy where a single consumption good is produced. The resource constraint is:

$$
\gamma c_{H}+(1-\gamma) c_{L}=y
$$

where $y$ is the aggregate production, and $c_{H}$ and $c_{L}$ are high-skilled and low-skilled consumption respectively.

Firms The available constant returns to scale technology can be represented through the following CES production function:

$y=F\left[\gamma\left(1-\ell_{H}\right),(1-\gamma)\left(1-\ell_{L}\right)\right]=\left[\phi\left[\gamma\left(1-\ell_{H}\right)\right]^{\delta}+\left[(1-\gamma)\left(1-\ell_{L}\right)\right]^{\delta}\right]^{1 / \delta}$,

where $\delta \in(0,1), \phi>1$, and $\left(1-\ell_{H}\right)$ and $\left(1-\ell_{L}\right)$ are high-skilled and lowskilled labor respectively. Firms are price takers and inverse labor demands 
are:

$$
\begin{aligned}
& \omega_{H}=\phi\left[\phi+\left(\frac{1-\gamma}{\gamma}\right)^{\delta}\left(\frac{1-\ell_{L}}{1-\ell_{H}}\right)^{\delta}\right]^{1 / \delta-1} \\
& \omega_{L}=\left[1+\phi\left(\frac{\gamma}{1-\gamma}\right)^{\delta}\left(\frac{1-\ell_{H}}{1-\ell_{L}}\right)^{\delta}\right]^{1 / \delta-1}
\end{aligned}
$$

Households Households in this economy derive utility from consumption and leisure. The utility function $U\left(c_{i}, \ell_{i}\right)$ is assumed to be strictly increasing and concave in both arguments. Households are endowed with one unit of time which can be devoted to work or leisure, and face the following budget constraint:

$$
c_{i}=\left(1-\tau_{i}\right) \omega_{i}\left(1-\ell_{i}\right)+T_{i}
$$

where $\omega_{i}$ is the wage, $\tau_{i}$ is the income tax rate and $T_{i}$ is a lump-sum transfer.

Government We consider two alternative policy schemes:

Policy scheme A The redistribution policy is implemented through a minimum wage law that sets a lower bound on low-skilled wages, $\omega_{\min }$, and an anonymous tax-transfer scheme $\{\tau, T\}$. The following government budget constraint holds:

$$
\gamma \tau \omega_{H}\left(1-\ell_{H}\right)+(1-\gamma) \tau \omega_{L}\left(1-\ell_{L}\right)=T
$$

Policy scheme B The redistribution policy is implemented through agent-specific tax rates $\left\{\tau_{H}, \tau_{L}\right\}$ and transfers $\left\{T_{H}, T_{L}\right\}$. The following government budget constraint holds:

$$
\gamma \tau_{H} \omega_{H}\left(1-\ell_{H}\right)+(1-\gamma) \tau_{L} \omega_{L}\left(1-\ell_{L}\right)=\gamma T_{H}+(1-\gamma) T_{L}
$$




\section{The equilibrium under alternative policies}

Equilibrium under policy scheme A Under policy scheme A wages must be higher than the legal lower bound, $\omega_{\min }$. Therefore, if the minimum wage is binding in equilibrium, there is an excess labor supply and the demand side determines labor allocations. Then, households face an additional restriction: there is a maximum number of hours, $\overline{S C}_{i}$, that they can allocate to work at the minimum wage $\omega_{\min } \cdot \overline{S C}_{i}$ is equal to the hours of $i$-skilled labor that the firm demands at the minimum wage in equilibrium.

Therefore, household $i$ 's decision problem is:

$$
\begin{aligned}
& \max _{\left\{c_{i}, \ell_{i}\right\}} U\left(c_{i}, \ell_{i}\right) \\
\text { s.t. } c_{i}= & (1-\tau) \omega_{i}\left(1-\ell_{i}\right)+T_{i} \\
\left(1-\ell_{i}\right) \leq & \overline{S C}_{i} \\
& \omega_{i}, \overline{S C}_{i}, \tau, T \text { given }
\end{aligned}
$$

The first order conditions for this problem are the consumer budget constraint (5) and the following inequalities:

$$
\begin{array}{rlll}
-U_{c}\left(c_{i}, \ell_{i}\right)(1-\tau) \omega_{i} & +U_{\ell}\left(c_{i}, \ell_{i}\right)+v_{i}=0 & \\
v_{i}\left[\overline{S C}_{i}-\left(1-\ell_{i}\right)\right] & =0 & \overline{S C}_{i}-\left(1-\ell_{i}\right) \geq 0 & v_{i} \geq 0
\end{array}
$$

When the minimum wage is binding, the multiplier $v_{i}$ is strictly positive and the $i$-skilled labor allocation is demand determined and equal to $\overline{S C}_{i}$. We consider economies where the minimum wage is only binding for low-skilled workers, i.e. high-skilled labor will never be constrained:

$$
\overline{S C}_{H}-\left(1-\ell_{H}\right)>0 \quad v_{L}=0
$$

In this economy, equilibria under policy scheme A are defined as follows:

Definition 1: Given a minimum wage $\omega_{\min }$, allocations $\left\{c_{H}, c_{L}, \ell_{H}, \ell_{L}\right\}$, a tax-transfer scheme $\{\tau, T\}$, wages $\left\{\omega_{H}, \omega_{L}\right\}$ and perceived constraints on the low-skilled labor supply $\overline{S C}_{L}$ constitute an equilibrium if the following conditions are satisfied: 
(i) $\left\{c_{H}, \ell_{H}\right\}$ solves the high-skilled household's decision problem given $\omega_{H}$ and policies $\{\tau, T\}$. That is,

$$
\begin{aligned}
-U_{c}\left(c_{H}, \ell_{H}\right)(1-\tau) \omega_{H}+U_{\ell}\left(c_{H}, \ell_{H}\right) & =0 \\
c_{H}=(1-\tau) \omega_{H}\left(1-\ell_{H}\right) & +T .
\end{aligned}
$$

(ii) $\left\{c_{L}, \ell_{L}\right\}$ solves the low-skilled household's decision problem given $\omega_{L}$ and policies $\{\tau, T\}$ and $\overline{S C}_{L}$. That is,

$$
\begin{aligned}
-U_{c}\left(c_{L}, \ell_{L}\right)(1-\tau) \omega_{L} & +U_{\ell}\left(c_{L}, \ell_{L}\right)+v_{L}=0 \\
\left(1-\ell_{L}\right) & =\overline{S C}_{L} \quad v_{L} \geq 0 \\
c_{L} & =(1-\tau) \omega_{L}\left(1-\ell_{L}\right)+T .
\end{aligned}
$$

(iii) $\left\{\ell_{H}, \ell_{L}\right\}$ maximizes firms' profits given $\omega_{H}$ and $\omega_{L}$. That is, $\left\{\ell_{H}, \ell_{L}\right\}$ satisfies (3) and (4).

(iv) The equilibrium wage $\omega_{L}$ is equal to or higher than $\omega_{\min }$. And $\overline{S C}_{L}$ is the quantity of low-skilled labor demanded at the minimum wage $\omega_{\min }$. That is,

$$
\omega_{L} \geq \omega_{\min }=F_{\ell_{L}}\left[\gamma\left(1-\ell_{H}\right),(1-\gamma) \overline{S C}_{L}\right]
$$

(v) The economy resource constraint (1) holds and the high-skilled labor market clears.

Equilibrium under policy scheme B Under policy scheme B there is no minimum wage legislation, although every household faces taxes and transfers that depend on her/his type.

Household $i$ 's problem is to maximize utility subject to the budget constraint (5), taking prices and policies as given. The first order conditions for this problem are the consumer budget constraint (5) and the following equation:

$$
-U_{c}\left(c_{i}, \ell_{i}\right)(1-\tau) \omega_{i}+U_{\ell}\left(c_{i}, \ell_{i}\right)=0
$$

Therefore equilibria under policy scheme B are defined as follows: 
Definition 2: Allocations $\left\{c_{H}, c_{L}, \ell_{H}, \ell_{L}\right\}$, taxes and transfers $\left\{\tau_{H}, \tau_{L}, T_{H}, T_{L}\right\}$ and wages $\left\{\omega_{H}, \omega_{L}\right\}$ constitute an equilibrium if the following conditions are satisfied:

(i) $\left\{c_{H}, \ell_{H}\right\}$ solves the high-skilled household's decision problem given $\omega_{H}$ and policies $\left\{\tau_{H}, T_{H}\right\}$. That is,

$$
\begin{aligned}
-U_{c}\left(c_{H}, \ell_{H}\right)\left(1-\tau_{H}\right) \omega_{H} & +U_{\ell}\left(c_{H}, \ell_{H}\right)=0 \\
c_{H} & =\left(1-\tau_{H}\right) \omega_{H}\left(1-\ell_{H}\right)+T_{H} .
\end{aligned}
$$

(ii) $\left\{c_{L}, \ell_{L}\right\}$ solves the low-skilled household's decision problem given $\omega_{L}$, policies $\left\{\tau_{L}, T_{L}\right\}$. That is,

$$
\begin{aligned}
-U_{c}\left(c_{L}, \ell_{L}\right)\left(1-\tau_{L}\right) \omega_{L} & +U_{\ell}\left(c_{L}, \ell_{L}\right)=0 \\
c_{L} & =\left(1-\tau_{L}\right) \omega_{L}\left(1-\ell_{L}\right)+T_{L} .
\end{aligned}
$$

(iii) $\left\{\ell_{H}, \ell_{L}\right\}$ maximizes firms' profits given $\omega_{H}$ and $\omega_{L}$. That is, $\left\{\ell_{H}, \ell_{L}\right\}$ satisfies (3) and (4).

(iv) All markets clear.

\section{The equivalence result}

In this section we show that for any equilibrium allocation under policy scheme A, a scheme B-type policy exists such that the same allocation is an equilibrium and vice versa.

Proposition 1: Let $\left\{\tau, T, \omega_{\min }\right\}$ be a policy under policy scheme A. Let allocation $\left\{c_{H}, c_{L}, \ell_{H}, \ell_{L}\right\}$ and wages $\left\{\omega_{H}, \omega_{L}\right\}$ constitute an equilibrium under $\left\{\tau, T, \omega_{\min }\right\}$. Consider a scheme B-type policy $\left\{\tau_{H}, \tau_{L}, T_{H}, T_{L}\right\}$ satisfying:

$T_{H}=T, \quad \tau_{H}=\tau, \quad \tau_{L}=1-\frac{U_{\ell}\left(c_{L}, \ell_{L}\right)}{\omega_{\min } U_{c}\left(c_{L}, \ell_{L}\right)}, \quad T_{L}=c_{L}-\left(1-\tau_{L}\right) \omega_{\min }\left(1-\ell_{L}\right)$. 
Then, $\left\{c_{H}, c_{L}, \ell_{H}, \ell_{L}\right\}$ and $\left\{\omega_{H}, \omega_{L}\right\}$ are also an equilibrium for the scheme $B$ type policy $\left\{\tau_{H}, \tau_{L}, T_{H}, T_{L}\right\}$.

Proof. We will prove that allocations $\left\{c_{H}, c_{L}, \ell_{H}, \ell_{L}\right\}$ fulfill equilibrium conditions under the agent-specific policy presented in the proposition. On the one hand, when $T_{H}=T$ and $\tau_{H}=\tau$, nothing changes in the constraint faced by high-skilled workers. Thus, the high-skilled household problem's first order conditions hold. On the other hand, $\tau_{L}$ is set in order for the marginal rate of substitution between consumption and leisure to equal the low-skilled after-tax wage and, therefore, make $v_{L}=0$; and $T_{L}$ is set to satisfy the low-skilled budget constraint. Hence, $\left\{\tau_{L}, T_{L}\right\}$ are such that $\left\{c_{L}, \ell_{L}\right\}$ maximizes the low-skilled household's utility given $\omega_{L}$. Finally, since allocations are the same under both policy schemes, the firms' problem first conditions and the resource constraint also hold with an agent-specific tax-transfer scheme $\left\{\tau_{H}, \tau_{L}, T_{H}, T_{L}\right\}$.

Proposition 2: Let the scheme B-type policy $\left\{\tau_{H}, \tau_{L}, T_{H}, T_{L}\right\}$ be such that,

$$
0>T_{H}-T_{L}=\left(\tau_{H}-\tau_{L}\right) \omega_{L}\left(1-\ell_{L}\right) .
$$

Let allocations $\left\{c_{H}, c_{L}, \ell_{H}, \ell_{L}\right\}$ and wages $\left\{\omega_{H}, \omega_{L}\right\}$ constitute an equilibrium under $\left\{\tau_{H}, \tau_{L}, T_{H}, T_{L}\right\}$. Consider a scheme $A$-type policy $\left\{\tau, T, \omega_{\min }\right\}$ satisfying:

$$
T=T_{H}, \quad \quad \tau=\tau_{H}, \quad \omega_{\min }=\omega_{L} .
$$

Then, $\left\{c_{H}, c_{L}, \ell_{H}, \ell_{L}\right\}$ and $\left\{\omega_{H}, \omega_{L}\right\}$ are also an equilibrium for the scheme A type policy $\left\{\tau, T, \omega_{\min }\right\}$.

Proof. As in the previous proof, it is straightforward that $\left\{c_{H}, c_{L}, \ell_{H}, \ell_{L}\right\}$ satisfy the firms' problem first conditions and the resource constraint for both policy schemes.

We now prove that allocations $\left\{c_{H}, c_{L}, \ell_{H}, \ell_{L}\right\}$ solve households' problems under the anonymous policy $\left\{\tau, T, \omega_{\min }\right\}$. Since $T=T_{H}$ and $\tau=\tau_{H}$, high-skilled households solve exactly the same problem under both policies. Thus, the high-skilled household's problem first order conditions hold for $\{\tau, T\}$. As regards the low-skilled workers, we have to prove first that 
the multiplier $v_{L}$ is positive; and then that $\left\{c_{L}, \ell_{L}\right\}$ satisfies their budget constraint under the new policy. The multiplier $v_{L}$ is such that

$$
v_{L}=U_{c}\left(c_{L}, \ell_{L}\right)(1-\tau) \omega_{L}-U_{\ell}\left(c_{L}, \ell_{L}\right)
$$

Since $\left\{c_{L}, \ell_{L}\right\}$ maximizes low-skilled utility for $\left\{\tau_{L}, T_{L}\right\}, U_{c}\left(c_{L}, \ell_{L}\right)(1-\tau) \omega_{L}-$ $U_{\ell}\left(c_{L}, \ell_{L}\right)=0$. Hence,

$$
v_{L}=U_{c}\left(c_{L}, \ell_{L}\right)(1-\tau) \omega_{L}-U_{c}\left(c_{L}, \ell_{L}\right)(1-\tau) \omega_{L}=\left(\tau_{L}-\tau\right) U_{c}\left(c_{L}, \ell_{L}\right) \omega_{L} .
$$

If, as assumed, $\tau=\tau_{H}<\tau_{L}$, then $v_{L}>0$.

Finally, we have to check that the low skilled budget constrained is satisfied. Assuming that $T_{H}-T_{L}=\left(\tau_{H}-\tau_{L}\right) \omega_{L}\left(1-\ell_{L}\right)$,

$c_{L}=(1-\tau) \omega_{L}\left(1-\ell_{L}\right)-T=\left(1-\tau_{H}\right) \omega_{L}\left(1-\ell_{L}\right)-\left(\tau_{H}-\tau_{L}\right) \omega_{L}\left(1-\ell_{L}\right)+T_{L}$.

Rearranging terms,

$$
c_{L}=\left(1-\tau_{L}\right) \omega_{L}\left(1-\ell_{L}\right)+T_{L}
$$

which is the low-skilled budget constraint when taxes and transfers are $\left\{\tau_{L}, T_{L}\right\}$.

\section{References}

Allen, S. (1987): "Taxes, Redistribution, and the Minimum Wage: A Theoretical Analysis", The Quarterly Journal of Economics, vol. August, pp 477-489.

Gorostiaga, A. and J.F. Rubio-Ramírez (2007): "Optimal Minimum Wages in a Competitive Economy: An Alternative Modelling Approach". Economic Modelling, vol. 24/5 pp. 778-796.

Guesnerie, R and K. Roberts (1987): "Minimum Wage Legislation as a Second Best Policy", European Economic Review, vol. 31, pp. 490-498. 